\title{
SUPPLEMENT TO EVALUATING EPOETIN DOSING STRATEGIES USING OBSERVATIONAL LONGITUDINAL DATA
}

\author{
By Cecilia A. Cotton and Patrick J. Heagerty
}

University of Waterloo and University of Washington

\section{APPENDIX A: ASYMPTOTICS OF CLONED IPW WEIGHTED LOG-RANK TEST}

To start we show that $W^{*}$ has the usual log-rank test statistic form:

$$
\begin{aligned}
W^{*} & =\sqrt{n} \int_{0}^{\infty} H(t)\left[d \hat{\Lambda}_{1}^{w}(t)-d \hat{\Lambda}_{2}^{w}(t)\right] \\
& =\sqrt{n} \int_{0}^{\infty} \frac{1}{n} \frac{Y_{1}^{w}(t) Y_{2}^{w}(t)}{Y_{1}^{w}(t)+Y_{2}^{w}(t)}\left[\frac{d N_{1}^{w}(t)}{Y_{1}^{w}(t)}-\frac{d N_{2}^{w}(t)}{Y_{2}^{w}(t)}\right] \\
& =\frac{1}{\sqrt{n}} \sum_{t=1}^{T} \frac{Y_{1}^{w}(t) Y_{2}^{w}(t)}{Y_{1}^{w}(t)+Y_{2}^{w}(t)}\left[\frac{d_{1}^{w}(t)}{Y_{1}^{w}(t)}-\frac{d_{2}^{w}(t)}{Y_{2}^{w}(t)}\right] \\
& =\frac{1}{\sqrt{n}} \sum_{t=1}^{T}\left[d_{1}^{w}(t)-Y_{1}^{w}(t)\left(\frac{d_{1}^{w}(t)+d_{2}^{w}(t)}{Y_{1}^{w}(t)+Y_{2}^{w}(t)}\right)\right]
\end{aligned}
$$

We will now prove that under $H_{0}, W^{*}$ is asymptotically normal with mean 0 and variance $\sigma^{2}$. Then we will find a consistent estimator for $\sigma^{2}$. Following notation similar to Equation (2) in Lee, Wei and Ying (1993) let $z_{i k}=I[k=1]$ and rewrite the test statistic $W^{*}$ as:

$$
W^{*}=\frac{1}{\sqrt{n}} \sum_{i=1}^{n} \sum_{k=1}^{2} \int_{0}^{\infty}\left[z_{i k}-\frac{W_{1 n}(t)}{W_{2 n}(t)}\right] d N_{i k}^{w}(t),
$$

where:

$$
\begin{aligned}
& W_{1 n}(t)=\frac{1}{n} \sum_{i=1}^{n} \sum_{k=1}^{2} z_{i k} w_{i k}(t) I\left(X_{i k} \leq t\right)=\frac{1}{n} Y_{1}^{w}(t) \\
& W_{2 n}(t)=\frac{1}{n} \sum_{i=1}^{n} \sum_{k=1}^{2} w_{i k}(t) I\left(X_{i k} \leq t\right)=\frac{1}{n}\left[Y_{1}^{w}(t)+Y_{2}^{w}(t)\right]
\end{aligned}
$$

Let $d M_{i k}^{w}=d N_{i k}^{w}(t)-Y_{i k}^{w} d \Lambda_{k}^{w}(t)$ and $d \bar{M}^{w}(t)=\sum_{i=1}^{n} \sum_{k=1}^{2} d M_{i k}^{w}$. Given the 
true weights $\Lambda_{k}^{w}(t)=\Lambda_{k}(t)$ so under $H_{0}: \Lambda_{1}^{w}(t)=\Lambda_{2}^{w}(t)$ it follows that:

$$
\begin{aligned}
W^{*} & =\frac{1}{\sqrt{n}} \sum_{i=1}^{n} \sum_{k=1}^{2} \int_{0}^{\infty}\left[z_{i k}-\frac{W_{1 n}(t)}{W_{2 n}(t)}\right] d M_{i k}^{w}(t) \\
& =\frac{1}{\sqrt{n}} \sum_{i=1}^{n} \sum_{k=1}^{2} \int_{0}^{\infty} z_{i k} d M_{i k}^{w}(t)-\frac{1}{\sqrt{n}} \int_{0}^{\infty} \frac{W_{1 n}(t)}{W_{2 n}(t)} d \bar{M}^{w}(t)
\end{aligned}
$$

Based on results for weighted martingales in Marzec and Marzec (1997) the processes:

$$
\psi_{k}(x)=\frac{1}{\sqrt{n}} \int_{0}^{x} d \sum_{i=1}^{n} M_{i k}^{w}(t)=\frac{1}{\sqrt{n}} \sum_{i=1}^{n} \int_{0}^{x} d M_{i k}^{w}(t)
$$

converge weakly in $(0, \tau)$, as $n \rightarrow \infty$ to a zero mean continuous Gaussian process $U_{k}(t), k=1,2$. Then following the same arguments given in Appendix A of Lee, Wei and Ying (1993) it follows that $W^{*}$ is asymptotically equivalent to:

$$
W^{* *}=\frac{1}{\sqrt{n}} \sum_{i=1}^{n} \sum_{k=1}^{2} \int_{0}^{\infty}\left[z_{i k}-\frac{w_{1}(t)}{w_{2}(t)}\right] d M_{i k}^{w}(t)
$$

where $w_{k}(t)$ are the limits of $W_{k n}(t)$.

Now let:

$$
\epsilon_{i}=\xi_{i 1}+\xi_{i 2} \quad \text { where } \quad \xi_{i k}=\int_{0}^{\infty}\left[z_{i k}-\frac{w_{1}(t)}{w_{2}(t)}\right] d M_{i k}^{w}(t)
$$

Since $w_{1}(t), w_{2}(t)$ are deterministic functions and $E\left[d M_{i k}^{w}(t)\right]=0$, it follows that $E\left[\epsilon_{i}\right]=0$ and $\operatorname{var}\left[\epsilon_{i}\right]=E\left[\epsilon_{i}^{2}\right]=\sigma^{2}$. In addition, subjects are assumed to be independent so $\epsilon_{1}, \ldots, \epsilon_{n}$ are independent random variables. Thus by the standard central limit theorem:

$$
W^{* *}=\frac{1}{\sqrt{n}} \sum_{i=1}^{n} \epsilon_{i} \rightarrow N\left(0, \sigma^{2}\right) \quad \text { where } \quad \sigma^{2}=\lim _{n \rightarrow \infty} \frac{1}{n} \sum_{i=1}^{n} \epsilon_{i}^{2}
$$

Recall that $d \hat{M}_{i k}^{w}(t)=d N_{i k}^{w}(t)-Y_{i k}^{w}(t) d \hat{\Lambda}^{w}(t)$ (Marzec and Marzec, 1997). Since $\hat{\Lambda}_{k}^{w}(t)$ is a consistent estimator of $\Lambda_{k}(t)$ (Robins and Finkelstein, 2000) a consistent estimate of $\sigma^{2}, \hat{\sigma}^{2}$, is obtained by using $\hat{\Lambda}_{k}^{w}(t)$ and replacing $w_{1}(t)$ and $w_{2}(t)$ with $W_{1 n}(t)=Y_{1}^{w}(t)$ and $W_{2 n}(t)=Y_{1}^{w}(t)+Y_{2}^{w}(t)$ respectively (Lee, Wei and Ying, 1993). Thus:

$\hat{\xi}_{i k}=\left[z_{i k}-\frac{Y_{1}^{w}(t)}{Y^{w}(t)}\right] w_{i k}\left(X_{i k}\right) \Delta_{i k}-\sum_{j=1}^{n} \sum_{l=1}^{2} \frac{Y_{i k}^{w}\left(X_{j l}\right)\left[z_{i k}-\frac{Y_{1}^{w}(t)}{Y^{w}(t)}\right] w_{j l}\left(X_{j l}\right) \Delta_{j l}}{Y^{w}\left(X_{j l}\right)}$ 


$$
\begin{gathered}
\hat{\epsilon}_{i}=\Delta_{i 1} w_{i 1}\left(X_{i 1}\right) \frac{Y_{2}^{w}\left(X_{i 1}\right)}{Y^{w}\left(X_{i 1}\right)}-\Delta_{i 2} w_{i 2}\left(X_{i 2}\right) \frac{Y_{2}^{w}\left(X_{i 2}\right)}{Y^{w}\left(X_{i 2}\right)} \\
-\sum_{j=1}^{n} \sum_{l=1}^{2} \frac{\Delta_{j l} w_{j l}\left(X_{j l}\right) Y_{i 1}^{w}\left(X_{j l}\right) Y_{2}^{w}\left(X_{j l}\right)}{\left[Y^{w}\left(X_{j l}\right)\right]^{2}}-\sum_{j=1}^{n} \sum_{l=1}^{2} \frac{\Delta_{j l} w_{j l}\left(X_{j l}\right) Y_{i 2}^{w}\left(X_{j l}\right) Y_{1}^{w}\left(X_{j l}\right)}{\left[Y^{w}\left(X_{j l}\right)\right]^{2}} \\
\hat{\sigma}^{2}=\frac{1}{n} \sum_{i=1}^{n} \hat{\epsilon}_{i}^{2}
\end{gathered}
$$

\section{APPENDIX B: SIMULATION DETAILS}

B.1. Rate Parameter Selection. The $\lambda_{k}$ exponential rate parameters need to be selected in such a way that their values persist through the cloning process. Let $p_{k}=n_{k} / n$ be the initial regimen allocation. For simplicity, assume an equal allocation and set $p=p_{k}=1 / K$ for all $k$. Consider the conditional probability of coadherence to regimen $j$ given adherence to $k$ as $p_{j \mid k}=P\left(A_{i j}(t)=1 \mid A_{i k}(t)=1\right)$. Note we assume the coadherence probabilities are the same at all time points and we will therefore drop $t$ from the notation. Use $\tilde{k}$ to represent the regimen under which a subject's survival time was generated. Assume $p_{j \mid k}=p_{k \mid j}$ or equivalently $p_{j k}=p_{j \mid k}=p_{k \mid j}$ where $p_{j k}=P\left(A_{i j}=1 \& A_{i k}=1\right)$. Fix $p_{j j}=1$. There are $\left(\begin{array}{c}K \\ 2\end{array}\right)$ coadherence probabilities $p_{j k}$ to be specified.

For the regimen under which a subject was generated, $P\left(A_{i k}=1 \mid \tilde{k}=\right.$ $k)=1$. So the overall probability of adherence to any particular regimen $j$ is:

$$
P\left(A_{i j}=1\right)=\sum_{l=1}^{K} P\left(A_{i j}=1 \mid \tilde{k}=l\right) P(\tilde{k}=l)=\sum_{l=1}^{K} p_{j \mid l} p_{l}=p \sum_{l=1}^{K} p_{j l}
$$

Using laws of conditional probability it follows that:

$$
P\left(\tilde{k}=k \mid A_{i j}=1\right)=\frac{P\left(A_{i j}=1 \mid \tilde{k}=k\right) P(\tilde{k}=k)}{P\left(A_{i j}=1\right)}=\frac{p_{j \mid k} p_{k}}{p \sum_{l=1}^{K} p_{j l}}=\frac{p_{j k}}{\sum_{l=1}^{K} p_{j l}}
$$

Let $\alpha_{k}=P\left(D_{i}=1 \mid \tilde{k}=k\right)$, the probability of death in the next time interval for any subject whose survival time was generated under regimen $k$. Note that $P\left(D_{i}=1 \mid \tilde{k}=k, A_{i j}=1\right)=P\left(D_{i}=1 \mid \tilde{k}=k\right), \forall j$ since the probability of death depends only on the initial generating regimen's rate and not on any coadherence. From this it follows that the probability of death in the next time interval given adherence to any regimen $j$ is:

$\alpha_{j}^{*}=P\left(D_{i}=1 \mid A_{i j}=1\right)=\sum_{k=1}^{K} P\left(D_{i}=1 \mid \tilde{k}=k, A_{i j}=1\right) P\left(\tilde{k}=k \mid A_{i j}=1\right)=\frac{\sum_{k=1}^{K} \alpha_{k} p_{j k}}{\sum_{l=1}^{K} p_{j l}}$ 
The probability of death in the next interval will persist through the cloning process if $\alpha_{k}=\alpha_{k}^{*}$. For the exponential distribution the probability of death in the next unit time interval is constant and $\alpha_{k}=1-\exp \left(-\lambda_{k}\right)$. Therefore $\alpha_{k}=\alpha_{k}^{*}$ is equivalent to $\lambda_{k}=\lambda_{k}^{*}$ where $\lambda_{k}^{*}=-\log \left(1-\alpha_{k}^{*}\right)$. Ideally all the $\lambda$ 's would persist through the cloning process. In other words $\lambda_{k}=\lambda_{k}^{*}, \lambda_{k}>0$, $\forall k$. After the specification of the $p_{j k}$ 's $\left(0<p_{j k}<1, \forall j, k\right)$ this yields $K$ equations with $K$ unknowns. Unfortunately the only solution to this system of equations is $\lambda_{1}=\lambda_{2}=\cdots=\lambda_{K}$ which is not particularly interesting.

Instead of requiring all the $\lambda_{k}=\lambda_{k}^{*}$ we chose to try and maintain only some of the hazard ratios through the cloning process. Specifically for $K=6$ we solve the following system of three equations:

$$
\frac{\lambda_{2}^{*}}{\lambda_{3}^{*}}=\frac{\lambda_{2}}{\lambda_{3}}, \frac{\lambda_{4}^{*}}{\lambda_{3}^{*}}=\frac{\lambda_{4}}{\lambda_{3}}, \frac{\lambda_{5}^{*}}{\lambda_{3}^{*}}=\frac{\lambda_{5}}{\lambda_{3}}
$$

for $\lambda_{2}, \lambda_{4}$ and $\lambda_{5}$. We do not require the hazards ratios for $k=1$ and $k=6$ to persist through the cloning process. That is, we allow $\frac{\lambda_{1}^{*}}{\lambda_{3}^{*}} \neq \frac{\lambda_{1}}{\lambda_{3}}$ and $\frac{\lambda_{6}^{*}}{\lambda_{3}^{*}} \neq \frac{\lambda_{6}}{\lambda_{3}}$. Calculations were done using the Maple programming language with coadherence probabilities $p_{j k}=1 / 2,1 / 3$ and $1 / 6$ for $|j-k|=1,2$ and $\geq 3$, respectively and fixed $\lambda_{1}=10, \lambda_{3}=5, \lambda_{6}=1$. This yields the solution $\lambda_{2}=5.515616827, \lambda_{4}=4.274687922$ and $\lambda_{5}=3.900982684$. Note that any constant multiple of all $\lambda_{k}$ is also a solution so the $\lambda_{k}$ can be scaled to become reasonable rate parameters for the exponential distribution. A scale factor of $1 / 100$ was applied to the above solution.

B.2. Introduction of Selective Nonadherence. At this stage we have built a system under which survival data can be generated in such a way that the true underlying causal hazard ratios are known and will persist through the cloning process under specified coadherence probabilities. The next step is to introduce selective nonadherence to induce a selection bias that can later be corrected for using IPW. In order to do this we include a baseline covariate associated with both survival and coadherence. A Copula model is used to generate pairs of random variables $\left(T_{i}, V_{i}\right)$ from a multivariate distribution with an exponential marginal distribution for $T_{i}$ and a $\operatorname{Binomial}\left(3, p_{1}=1 / 3, p_{2}=1 / 3\right)$ marginal distribution for $V_{i}$. Lower values of $V_{i}$ are associated with lower values of $T_{i}$. Three scenarios are considered with copula parameters $\alpha=0,0.5,2$ to induce no, moderate, and severe selection bias, respectively.

To induce selective nonadherence, the coadherence probabilities used in the cloning process are modified conditional on $V_{i}$. Coadherence probabilities for a subject generated under regimen $\tilde{k}$ and when being cloned under 
TABLE 1

Coadherence probabilities used to induce selective nonadherence in cloning simulations for subjects generated under original regimen $\tilde{k}$ and cloned under regimen $j$ with baseline covariate $V$

\begin{tabular}{cccc}
\hline \hline & $V=0 \& j=1,2,3$ & & $V=0 \& j=4,5,6$ \\
& $V=2 \& j=4,5,6$ & $V=1$, all $j$ & $V=2 \& j=1,2,3$ \\
\hline$|j-\tilde{k}|=1$ & $13 / 18$ & $1 / 2$ & $5 / 18$ \\
$|j-\tilde{k}|=2$ & $1 / 2$ & $1 / 3$ & $1 / 6$ \\
$|j-\tilde{k}| \geq 3$ & $5 / 18$ & $1 / 6$ & $1 / 18$ \\
\hline
\end{tabular}

regimen $j$ are given in Table 1. Using the notation of Appendix B.1 these are $P\left(A_{i j}=1 \mid \tilde{k}=k, V_{i}=v\right)$. When $j=1,2,3$, subjects with low $V_{i}$ are more likely to be coadherent with other regimens. Conversely, for $j=4,5,6$, subjects with high $V_{i}$ values are more likely to be coadherent with other regimens. In all cases, subjects are more likely to be adherent to regimen $j$ if $|j-\tilde{k}|$ is small. Note that the selection of the conditional probabilities was subject to the original unconditional probabilities used to generate the $\lambda$ 's. Since $P\left(V_{i}=0\right)=P\left(V_{i}=1\right)=P\left(V_{i}=2\right)$ this restriction is equivalent to the row means in Table 1 equaling the probabilities given in Appendix B.1.

Under the specified $\lambda_{k}$ values, survival is higher for larger values of $k$. Therefore this selective nonadherence means that subjects with larger $V_{i}$ values (and therefore larger $T_{i}$ values) are more likely to be adherent to regimens with longer mean survival. This will have the effect of overestimating the probability of survival for these regimens. Put simply, the adherent subjects (those included in the analysis set) will tend to have longer survival times than the nonadherent/censored subjects.

\section{REFERENCES}

LeE, E. W., Wei, L. J. and Ying, Z. (1993). Linear regression analysis for highly stratified failure time data. Journal of the American Statistical Association 88 557-565.

Marzec, L. and Marzec, P. (1997). Generalized martingale-residual processes for goodness-of-fit inference in Cox's type regression models. The Annals of Statistics $\mathbf{2 5}$ $683-714$.

Robins, J. M. and Finkelstein, D. M. (2000). Correcting for noncompliance and dependent censoring in an AIDS clinical trial with inverse probability of censoring weighted (IPCW) log-rank tests. Biometrics 56 779-788.

C.A. Cotton

Department of Statistics and Actuarial Science

UNIVERSITY OF WATERLOO

200 University Avenue West

Waterloo, Ontario N2L 3G1

CANADA

E-MAIL: ccotton@uwaterloo.ca
P.J. Heagerty

Department of Biostatistics

UNIVERSITY OF WASHINGTON

Seattle, WA 98195

USA

E-MAIL: heagerty@u.washington.edu 